\title{
Tonicity of human tear fluid sampled from the cul-de-sac
}

\author{
WILLIAM J BENJAMIN AND RICHARD M HILL \\ From the University of Alabama at Birmingham, Birmingham, Alabama, USA, and the Ohio State \\ University, Columbus, Ohio, USA
}

SUMmaRY A 'freezing point' depression technique was used to determine the osmolality of 384 samples of tear fluid originating from the middle of the lower tear prism and the lower cul-de-sac of one eye of each of 12 young adults. Tear fluid from the cul-de-sac (mean $341.0 \mathrm{mosm} / \mathrm{kg}$ ) was found overall to be significantly hypertonic $(\mathrm{p}<0.0001)$ relatively to fluid from the tear prism (mean $315 \cdot 5$ $\operatorname{mosm} / \mathrm{kg}$ ). However, the difference between the two sampling sites was highly variable between individuals, ranging from a maximum mean site difference of $64 \cdot 5$. $\mathrm{mosm} / \mathrm{kg}$ for one of the six culde-sacs found to be significantly hypertonic, to a mean site difference of $25.0 \mathrm{mosm} / \mathrm{kg}$ for one of the two cul-de-sacs found to be significantly hypotonic. These results suggest that a unique localised tear environment exists inside the lower cul-de-sac, which has several clinical consequences - for example, for pharmaceutical absorption, ocular microbiology, and hydrophilic contact lens performance.

Temporal and spatial variations in the osmolality of human tear fluid have been observed across the ocular surface by sampling of tear fluid in amounts of approximately $0 \cdot 2 \mu \mathrm{l}$ from the mid point of the lower tear prism ${ }^{\prime 2}$ and also from tear prism sites at other locations. ${ }^{3}$ While the surface tear fluid has been shown in these studies to be osmotically heterogeneous in the open-eye state, it is not clear to what degree and in which direction of magnitude the tear fluids within less exposed parts of the ocular adnexa (that is, within the cul-de-sacs) may vary from fluid at the surface of the eye.

The routes and rates by which tear fluids in the culde-sacs are turned over have received little experimental attention but must be assumed to be different and slower, respectively, than the normal flow of surface tear prisms owing to probable 'entrapment' of fluid inside the fornices. The purpose of the present study was to test for osmotic differences between tear fluids sampled from the lower tear prism, being the reference point from which most previous work has been performed, and the lower cul-de-sac, from which samples could also be taken without stimulation of reflex tearing.

Correspondence to Dr William J Benjamin, University of Alabama at Birmingham, School of Optometry/The Medical Center, University Station, Birmingham, Alabama 35294, USA.

\section{Material and methods}

Tear fluid osmolality determinations were made with a 'freezing point' osmometer capable of at least $1 \%$ accuracy with fluid samples of approximately $0 \cdot 2$ $\mu l .{ }^{14}$ The experimental technique was based on the measurement of melting point of tear samples and its relationship to tear osmolality. A pilot study was first attempted by sampling with finely drawn microcapillary tubes the tear fluid at the middle of the lower tear prism and then from the lower cul-de-sac of the left eye of each of two young adults. While subject 2 was asymptomatic, subject 1 had occasional complaints of dry eye, though no ocular surface staining with rose Bengal was biomicroscopically noted (nor was staining noted on any subject).

Tear samples were each immediately loaded into oil and simultaneously processed with a standard calibration liquid, ' a technique confirmed by Martin' to give repeatable results realistic to the tonicity of human tear fluid. ${ }^{6}$ After analysis of the preliminary data indicated that an osmotic difference between the two sampling positions was probable, an expanded series of osmolality determinations was performed for the two sampling sites of each subject. A total of 184 osmolality determinations resulted from the processing of these initial samples. 
Ten additional asymptomatic young adult subjects were then added to the study, bringing the mean age of the 12 subjects in the whole study to $25 \cdot 3$ years (range 22 to 32 years). Each of the 10 additional subjects submitted 10 pairs of tear samples (10 samples from the lower tear prism and 10 samples from the cul-de-sac), every pair of samples consisting first of fluid taken from the lower tear prism and then also from the cul-de-sac. Sample pairs were taken at least 15 minutes apart, and osmolality determinations for both tear fluids of each pair of samples were made simultaneously with the standard calibration liquid.

\section{Results}

An analysis of variance ${ }^{7}$ performed unweighted with respect to the additional number of measurements recorded for subjects 1 and $2(F(1,360)=37 \cdot 58$, $\mathrm{p}<0.0001)$ revealed that, for the population, the mean osmolality of tear fluid taken from the cul-desac $(341.0 \mathrm{mosm} / \mathrm{kg})$ was significantly higher than that taken from the lower tear prism $(315.5$ mosm/ $\mathrm{kg}$ ). The main effect of subject was significant $(\mathrm{F}(11,360)=15 \cdot 52, \mathrm{p}<0 \cdot 0001)$, as was also the interaction between subject and sampling location $(F(11,360)=2 \cdot 74, p<0 \cdot 0021)$.

The mean tear fluid osmolalities with standard deviations for the two sampling sites of each subject and of the population are shown in Table 1. Analyses of variance were computed from the data from each subject alone and from the population (as noted above); the corresponding critical statistics are shown in Table 1. Single asterisks have been placed

Table 1 Mean tear fluid osmolalities in mosm/kg ( $S D$ in parentheses) for samples taken from the lower tear prism and cul-de-sac of 12 subjects. $F$ values and probability levels for the differences between site means of each subject, and of the population, are also shown

\begin{tabular}{|c|c|c|c|c|c|c|}
\hline Subject & $\begin{array}{l}\text { Mean } \\
\text { lower prism }\end{array}$ & $n$ & $\begin{array}{l}\text { Mean } \\
\text { cul-de-sac }\end{array}$ & $n$ & $F(d f 1, d f 2)$ & $p$ Value \\
\hline 1 & $344 \cdot 0(61 \cdot 7)$ & 51 & $408 \cdot 5(57 \cdot 6)$ & 32 & $22 \cdot 53(1,81)$ & $0 \cdot\left(0001^{*}\right.$ \\
\hline 2 & $326 \cdot 5(50 \cdot 3)$ & 57 & $370 \cdot 0(65 \cdot 4)$ & 44 & $14 \cdot 37(1,99)$ & $0 \cdot\left(0003^{*}\right.$ \\
\hline 3 & $304 \cdot 0(13 \cdot 1)$ & 10 & $280 \cdot 5(14 \cdot 8)$ & 10 & $14 \cdot 15(1,18)$ & $0.0014^{*}$ \\
\hline 4 & $311 \cdot 0(19.7)$ & 10 & $330 \cdot 5(24 \cdot 3)$ & 10 & $3 \cdot 88(1,18)$ & $0.0643^{* *}$ \\
\hline 5 & $323.5(42 \cdot 8)$ & 10 & $314 \cdot 0(36 \cdot 5)$ & 10 & $0 \cdot 29(1,18)$ & 0.5999 \\
\hline 6 & $327 \cdot 0(35 \cdot 8)$ & 10 & $302 \cdot 0(11 \cdot 1)$ & 10 & $4.46(1,18)$ & $0 \cdot(0490 *$ \\
\hline 7 & $293 \cdot 0(43 \cdot 7)$ & 10 & $337.5(26.4)$ & 10 & $7 \cdot 59(1,18)$ & $0 \cdot(0130 *$ \\
\hline 8 & $277 \cdot()(39 \cdot 8)$ & 10 & $299 \cdot 0(30 \cdot 7)$ & 10 & $1.91(1,18)$ & $0 \cdot 1834$ \\
\hline 9 & $244 \cdot 0(56 \cdot 0)$ & 10 & $253.0(49.9)$ & 10 & $0 \cdot 14(1,18)$ & 0.7088 \\
\hline 10 & $297 \cdot 0(40 \cdot 4)$ & 10 & $303.0(55.9)$ & 10 & $0.08(1,18)$ & 0.7864 \\
\hline 11 & $267 \cdot 0(51 \cdot 7)$ & 10 & $308 \cdot 0(52.9)$ & 10 & $3.08(1,18)$ & $0.0964 * *$ \\
\hline 12 & $306 \cdot 0(16 \cdot 8)$ & 10 & $337 \cdot 5(21 \cdot 6)$ & 10 & $13 \cdot 07(1,18)$ & $0.0020 *$ \\
\hline All & $315 \cdot 5(54 \cdot 6)$ & 208 & $341 \cdot 0(66 \cdot 1)$ & 176 & $37 \cdot 58(1,360)$ & $0 \cdot\left(0001^{*}\right.$ \\
\hline
\end{tabular}

*Comparisons significant to at least the $0 \cdot 05$ level.

**Comparisons significant to at least the $0 \cdot 10$ level. next to the statistics of those subjects for which the mean tear fluid osmolalities of the two sampling sites were found to be significantly different to at least the 0.05 level. Two asterisks have been placed next to those statistics indicating significance to at least the $0 \cdot 10$ level. The mean osmolalities for each subject are plotted in Fig. 1, and a linear correlation between the mean cul-de-sac and tear prism values of the 12 subjects shown in Table 1 (12 pairs of mean data points) resulted in a correlation coefficient of $+0 \cdot 72$.

\section{Discussion}

Osmotic variations of human tear fluid have been reported between several collection sites at the tear fluid prisms surrounding the exposed ocular surface. Tear fluid at these sites may be vulnerable to environmental influences, such as ambient humidity, temperature, and flow of air across the surface of the eye, in addition to the effects of pollutants and reflex tearing. Less vulnerable to outside influences, perhaps, might be tear fluid within the cul-de-sac, where the immediate environment may be more stable as in a more 'closed' system.

In comparison with the osmolality of tear fluid sampled from the lower tear prism, a standard site from which tear osmolality determinations have been routinely made, the fluid from the lower cul-de-sac was generally hypertonic over the population of subjects shown in Table 1 and Fig. 1. Tear fluid from the lower cul-de-sac was $8 \%(25.5 \mathrm{mosm} / \mathrm{kg})$ more hypertonic, on average, than that of the lower tear prism established in rooms averaging $23^{\circ} \mathrm{C}$ and $43 \%$ relative humidity. This site difference should be considered on the conservative side, because on opening of the lower cul-de-sac for sampling some mixture from the lower tear prism could have occurred, thus possibly reducing the average tonicity of fluid sampled from the cul-de-sac.

The subject means for the two collection sites were positively correlated (Fig. 1), with a correlation coefficient of +0.72 , and statistically significant differences between the mean tonicities of the two collection sites were found for 8 of the 12 subjects. The site difference was greatest for subject 1 (the 'dry eye' patient who also had the highest tear fluid tonicities), for whom the cul-de-sac fluid was $408 \cdot 5$ $\mathrm{mosm} / \mathrm{kg}$, or $19 \%(64.5 \mathrm{mosm} / \mathrm{kg})$ more hypertonic than fluid from the lower tear prism. Relatively hypertonic cul-de-sac fluid was statistically supportable for six of the subjects (subjects 1,2, 4, 7, 11, and 12), the cul-de-sac osmolality for these subjects ranging as low as $308.0 \mathrm{mosm} / \mathrm{kg}$ for subject 11 . The minimum statistically significant site difference was $6 \%(19.5 \mathrm{mosm} / \mathrm{kg})$ in the case of subject 4 , for which 


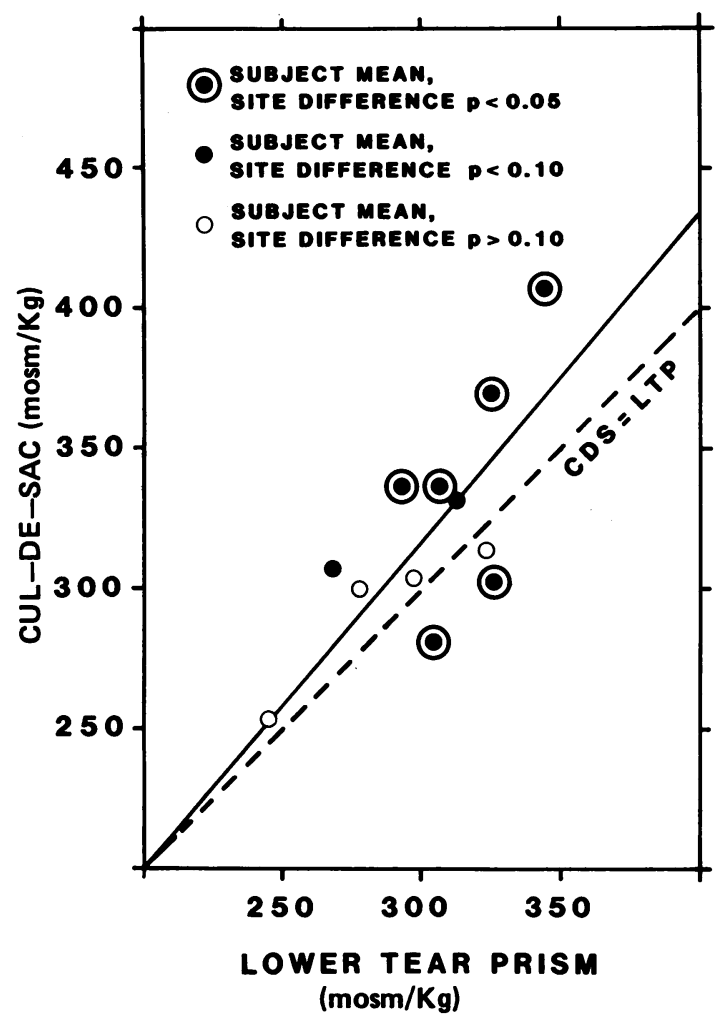

Fig. 1 The mean tear fluid osmolalities for the lower cul-desac (CDS) and lower tear prism (LTP) of each of the 12 subjects have been plotted. An open circle represents a site comparison in which cul-de-sac and lower tear prism mean values were not significantly different. A filled circle represents a site comparison significant to at least the $0 \cdot 10$ level, while a filled circle surrounded by an annulus represents a site difference significant to at least the 0.05 level. The dashed line indicates where osmolalities of the two sites would be equal (CDS $=L T P)$, produced for comparison with the actual mean data points and the solid linear regression line for those points, which had a correlation coreficient of $+0 \cdot 72$.

the cul-de-sac fluid was more hypertonic than the tear prism average.

A reversed relationship was found for two subjects, in that hypotonicity of the cul-de-sac fluid was statistically supportable relative to tear fluid of the lower tear prism. The cul-de-sac fluid was $8 \%$ hypotonic for subjects 3 and 6 (site differences of 23.5 and $25.0 \mathrm{mosm} / \mathrm{kg}$, respectively, in Table 1). Site differences were not statistically significant for subjects 5 , 8,9 , and 10 .

Cul-de-sac tear fluid therefore has a unique osmotic environment, tending to hypertonicity in most cases relative to the tear fluid tonicity of the lower tear prism. One might have expected, how- ever, a general hypotonicity due to reduced evaporation of cul-de-sac fluid relative to tear prism fluid exposed to air, as did in fact occur for subjects 3 and 6. This hypothesis has been offered earlier for another 'closed' tear environment, that being the hypotonic shift of tear prism fluid found after sleep. ${ }^{8}$

More likely for the six subjects with hypertonic culde-sac fluids, however, are the effects of a possible reduction of aqueous tear circulation within the culde-sac area in comparison with the ocular surface. Accounts of tear fluid elimination ${ }^{4-11}$ are not necessarily inclusive of fluids within the cul-de-sacs, and the efficiency of the elimination routes may vary between individuals. In addition, basic and reflex aqueous tear fluids primarily originate from structures of the upper eyelid and are possibly less prone to replenish the compartment of the lower cul-de-sac. It is therefore plausible that tear fluid within the lower cul-de-sac is less stirred than fluid from the ocular surface, with higher accumulations of ionic and other small-particle by-products from enzymatic breakdown of mucous strands ${ }^{91213}$ and from the surrounding epithelial cell layers. Even for the four subjects not showing statistically significant site differences the competing effects of stagnation and reduced evaporation on tonicity within the cul-de-sac may have offsetting each other.

The presence of unique tear fluid 'compartments' within the lower cul-de-sac of individuals raises many questions related to future clinical studies. Could these tear compartments be more reliable indicators of incipient dry eye, being generally more hypertonic and perhaps less alterable by outside elements, than their ocular surface counterparts? Are not epithelial lesions in dry eye the cause of heightened tear osmolality (and not vice versa), given that human epithelial cells in the cul-de-sac seem to withstand a much higher tear fluid osmolality in some subjects than previously thought? Could disturbances of the cul-de-sac fluid have more effect on the stability of the ocular and palpebral surfaces during contact lens wear or on pathological states than previously recognised, since this fluid interacts with a more expanded and vascularised tissue than does the precorneal tear film? In addition, tear fluid osmolality may indicate the effectiveness of tear elimination in the lower culde-sac, and it may influence microbiological distributions around the eye as well as the hydration, retention, and drug delivery characteristics of various types of ocular inserts.

\section{References}

1 Benjamin WJ, Hill RM. Human tears: osmotic characteristics. Invest Ophthalmol Vis Sci 1983; 24: 1624-6.

2 Benjamin WJ, Armitage BS, Woloschak MJ, Hill RM. Nanoliter tracking of the tears. J Am Optom Assoc 1983; 54: 243-4. 
3 Benjamin WJ, Hill RM. Tear osmotic differences across the ocular surface. Graefes Arch Clin Exp Ophthalmol 1986: 224: $583-6$.

4 Gilbard JP, Farris RL, Santamaria J II. Osmolarity of tear microvolumes in keratoconjunctivitis sicca. Arch Ophthalmol 1978: 96: 677-81.

5 Martin DK. Osmolality of the tear fluid in the contralateral eye during monocular contact lens wear. Acta Ophthalmol (Khh) 1987; 65: 551-5.

6 Benjamin WJ, Hill RM. Statistical evaluation of tear fluid osmolality measurements. Invest Ophthalmol Vis Sci (ARVO suppl) 1988: 29: 278.

7 SAS User's Guide: Statistics, Version 5 Edition. North Carolina: SAS Institute, Box 8000, Cary, 1985: 470-6.

8 Terry JE, Hill RM. Human tear osmotic pressure: diurnal variations and the closed eye. Arch Ophthalmol 1978; 96: 120-2.
9 Norn MS. Mucus flow in the conjunctiva. Rate of migration of the mucous thread in the inferior conjunctival fornix towards the inner canthus. Acta Ophthalmol (Khh) 1969; 47: 129-46.

10 Doane MG. Interaction of eyelids and tears in corneal wetting and the dynamics of the normal human blink. Am J Ophthalmol 1980; 89: 507-16.

11 Lemp MA, Weiler HH. How do tears exit? Invest Ophthalmol Vis Sci 1983; 24: 619-22.

12 Norn MS. Birefringence of mucous fibrils in the mucous thread of the inferior conjunctival fornix. Acta Ophthalmol (Khh) 1969: 47: 723-34.

13 Holly FJ, Lemp MA. Tear physiology and dry eyes. Surv Ophthalmol 1977; 22: 69-87.

Accepted for publication 6 January 1989. 